\title{
HOMOTOPY PERTURBATION ALGORITHM USING LAPLACE TRANSFORM FOR GAS DYNAMICS EQUATION
}

\author{
JAGDEV SINGH, DEVENDRA KUMAR AND SUSHILA
}

\begin{abstract}
In this paper, we apply a combined form of the Laplace transform method with the homotopy perturbation method to obtain the solution of nonlinear gas dynamics equation. This method is called the homotopy perturbation transform method (HPTM). This technique finds the solution without any discretization or restrictive assumptions and avoids the round-off errors. The fact that this scheme solves nonlinear problems without using Adomian's polynomials can be considered as a clear advantage of this algorithm over the decomposition method. The results reveal that the homotopy perturbation transform method (HPTM) is very efficient, simple and can be applied to other nonlinear problems.
\end{abstract}

Mathematics Subject Classification 2000: 39B12, 35F25

Key Words and Phrases: Laplace transform method, Homotopy perturbation method, Gas dynamics equation, He's Polynomials

\section{INTRODUCTION}

Nonlinear phenomena play a crucial role in applied mathematics and physics. We know that most of engineering problems are non-linear, and it is difficult to solve them analytically. The importance of obtaining the exact or approximate solutions of nonlinear partial differential equations in physics and mathematics is still a significant problem that needs new methods to discover exact or approximate solutions. Various powerful mathematical methods have been proposed for obtaining exact and approximate analytic solutions. Some of the classic analytic methods are Lyapunov's artificial small parameter method [1], perturbation techniques [2-4], $\delta$-expansion method [5] and Hirota bilinear method $[6,7]$. In recent years, many research workers have paid attention to study the solutions of nonlinear partial differential equations by using various methods. Among these are the Adomian decomposition method (ADM) [8], He's semi-inverse method [9], the tanh method, the homotopy perturbation method (HPM), the differential transform method and the variational iteration method (VIM) [10-17]. He [25-38] developed the homotopy perturbation method (HPM) by merging the standard homotopy and perturbation for solving various physical problems. It is worth mentioning that the HPM is applied without any discretization, restrictive assumption or transformation and is free from round off errors. The Laplace transform is totally incapable of handling nonlinear equations because of the difficulties that are caused by the nonlinear terms. Various ways have been proposed recently to deal with these nonlinearities such as the Adomian decomposition method [39] and the Laplace decomposition algorithm [40-44]. Furthermore, the homotopy perturbation method is also combined with the well-known Laplace transformation method [45] and the variational iteration method [47] to produce a highly effective technique for handling many nonlinear problems. In a recent paper 
Khan and $\mathrm{Wu}$ [23] proposed the homotopy perturbation transform method (HPTM) for solving the nonlinear equations. It is worth mentioning that the HPTM is an elegant combination of the Laplace transformation, the homotopy perturbation method and He's polynomials and is mainly due to Ghorbani [20, 21]. The homotopy perturbation transform method (HPTM) provides the solution in a rapid convergent series which may lead to the solution in a closed form. The advantage of this method is its capability of combining two powerful methods for obtaining exact solutions for nonlinear equations. Recently several techniques including the Adomian decomposition method and the homotopy perturbation method (HPM) have been used to handle nonlinear homogeneous gas dynamics equations $[18,19]$. Inspired and motivated by the ongoing research in this area, we use the homotopy perturbation transform method (HPTM) in solving the following nonlinear homogeneous gas dynamics equation

$$
\frac{\partial u}{\partial t}+\frac{1}{2}\left(u^{2}\right)_{x}-u(1-u)=0, \quad 0 \leq x \leq 1, t>0,
$$

with the initial condition

$$
u(x, 0)=g(x) .
$$

\section{HOMOTOPY PERTURBATION TRANSFORM METHOD (HPTM)}

To illustrate the basic idea of this method, we consider a general nonlinear partial differential equation with the initial conditions of the form:

$$
\begin{aligned}
& D u(x, t)+R u(x, t)+N u(x, t)=g(x, t), \\
& u(x, 0)=h(x), u_{t}(x, 0)=f(x),
\end{aligned}
$$

where $\mathrm{D}$ is the second order linear differential operator $D=\partial^{2} / \partial t^{2}, \mathrm{R}$ is the linear differential operator of less order than $\mathrm{D}, \mathrm{N}$ represents the general nonlinear differential operator and $\mathrm{g}(\mathrm{x}, \mathrm{t})$ is the source term. Taking the Laplace transform (denoted in this paper by $L$ ) on both sides of eq. (3), we get

$$
L[D u(x, t)]+L[R u(x, t)]+L[N u(x, t)]=L[g(x, t)] .
$$

Using the differentiation property of the Laplace transform, we have

$$
L[u(x, t)]=\frac{h(x)}{s}+\frac{f(x)}{s^{2}}-\frac{1}{s^{2}} L[R u(x, t)]+\frac{1}{s^{2}} L[g(x, t)]-\frac{1}{s^{2}} L[N u(x, t)] .
$$

Operating with the Laplace inverse on both sides of eq. (5) gives

$$
u(x, t)=G(x, t)-L^{-1}\left[\frac{1}{s^{2}} L[R u(x, t)+N u(x, t)]\right],
$$

where $G(x, t)$ represents the term arising from the source term and the prescribed initial conditions. Now we apply the homotopy perturbation method

$$
u(x, t)=\sum_{n=0}^{\infty} p^{n} u_{n}(x, t)
$$

and the nonlinear term can be decomposed as 
HOMOTOPY PERTURB. ALGORITHM USING LAPLACE TRANSFORM ...

$$
N u(x, t)=\sum_{n=0}^{\infty} p^{n} H_{n}(u)
$$

for some He's polynomials $H_{n}(u)($ see $[45,46])$ that are given by

$$
H_{n}\left(u_{0}, u_{1}, \ldots, u_{n}\right)=\frac{1}{n !} \frac{\partial^{n}}{\partial p^{n}}\left[N\left(\sum_{i=0}^{\infty} p^{i} u_{i}\right)\right]_{p=0}, \mathrm{n}=0,1,2,3 \ldots
$$

Substituting eq. (8) and eq. (7) in eq. (6), we get

$$
\sum_{n=0}^{\infty} p^{n} u_{n}(x, t)=G(x, t)-p\left(L^{-1}\left[\frac{1}{s^{2}} L\left[R \sum_{n=0}^{\infty} p^{n} u_{n}(x, t)+\sum_{n=0}^{\infty} p^{n} H_{n}(u)\right]\right)\right)
$$

which is the coupling of the Laplace transform and the homotopy perturbation method using He's polynomials. Comparing the coefficient of like powers of $p$, the following approximations are obtained.

$$
\begin{aligned}
& p^{0}: u_{0}(x, t)=G(x, t) \\
& p^{1}: u_{1}(x, t)=-L^{-1}\left[\frac{1}{s^{2}} L\left[R u_{0}(x, t)+H_{0}(u)\right]\right], \\
& p^{2}: u_{2}(x, t)=-L^{-1}\left[\frac{1}{s^{2}} L\left[R u_{1}(x, t)+H_{1}(u)\right]\right], \\
& p^{2}: u_{2}(x, t)=-L^{-1}\left[\frac{1}{s^{2}} L\left[R u_{2}(x, t)+H_{2}(u)\right]\right],
\end{aligned}
$$

\section{EXPERIMENTAL EVALUATION}

In this section we consider the following nonlinear homogeneous gas dynamics equation

$$
\frac{\partial u}{\partial t}+\frac{1}{2}\left(u^{2}\right)_{x}-u(1-u)=0, \quad 0 \leq x \leq 1, t>0,
$$

with the initial condition

$$
u(x, 0)=g(x)=e^{-x} .
$$

Taking the Laplace transform on both sides of eq. (12) subject to the initial condition (13), we have 


$$
L[u(x, t)]=\frac{e^{-x}}{s}-\frac{1}{2 s} L\left[\left(u^{2}\right)_{x}\right]+\frac{1}{s} L[u(1-u)]
$$

The inverse of Laplace transform implies that

$$
u(x, t)=e^{-x}-L^{-1}\left[\frac{1}{s} L\left[\frac{1}{2}\left(u^{2}\right)_{x}-u(1-u)\right]\right]
$$

Now, applying the homotopy perturbation method, we get

$$
\begin{aligned}
\sum_{n=0}^{\infty} p^{n} u_{n}(x, t)=e^{-x}-p & {\left[L ^ { - 1 } \left[\frac { 1 } { s } L \left[\frac{1}{2}\left(\left(\sum_{n=0}^{\infty} p^{n} u_{n}(x, t)\right)^{2}\right)_{x}\right.\right.\right.} \\
& \left.\left.\left.-\sum_{n=0}^{\infty} p^{n} u_{n}(x, t)\left(1-\sum_{n=0}^{\infty} p^{n} u_{n}(x, t)\right)\right]\right]\right)
\end{aligned}
$$

Comparing the coefficients of like powers of $\mathrm{p}$, we have

$$
\begin{aligned}
& p^{0}: u_{0}(x, t)=e^{-x} \\
& p^{1}: u_{1}(x, t)=-L^{-1}\left[\frac{1}{s} L\left[\frac{1}{2} u_{0 x}^{2}-u_{0}\left(1-u_{0}\right)\right]\right]=e^{-x} t,
\end{aligned}
$$

$$
p^{2}: u_{2}(x, t)=-L^{-1}\left[\frac{1}{s} L\left[\frac{1}{2}\left(u_{0} u_{1}\right)_{x}-u_{1}+u_{0} u_{1}\right]\right]=e^{-x} \frac{t^{2}}{2} .
$$

Proceeding in a similar manner, we have

$$
\begin{aligned}
& p^{3}: u_{3}(x, t)=e^{-x} \frac{t^{3}}{6}, \\
& \vdots
\end{aligned}
$$

Therefore the solution $u(x, t)$ is given by

$$
u(x, t)=e^{-x}\left(1+t+\frac{1}{2} t^{2}+\frac{1}{6} t^{3}+\cdots\right)=e^{t-x} .
$$

It is obvious that a higher number of iterations make $u_{n}(x, t)$ converge to the exact solution $e^{t-x}$. 


\section{CONCLUSIONS}

In this paper, the homotopy perturbation transform method (HPTM) was successfully applied to study the homogeneous case of nonlinear gas dynamics with initial condition. The results show that the homotopy perturbation transform method (HPTM) is powerful and efficient technique in finding exact and approximate solutions for nonlinear differential equations. It is worth mentioning that HPTM is capable of reducing the volume of the computational work as compared to the classical methods while still maintaining the high accuracy of the numerical result; the size reduction amounts to an improvement of the performance of the approach. The fact that the HPTM solves nonlinear problems without using Adomian's polynomials is a clear advantage of this technique over the decomposition method. In conclusion, the HPTM may be considered as a nice refinement in existing numerical techniques and might find the wide applications.

\section{ACKNOWLEDGMENTS}

The authors are grateful to the referees for their invaluable suggestions and comments for the improvement of the paper.

\section{REFERENCES}

[1] A.M. Lyapunov, The General Problem of the Stability of Motion, Taylor \& Francis, London, UK, 1992, English translation.

[2] J.H. He, Homotopy perturbation technique, Computer Methods in Applied Mechanics and Engineering, 178 (1999), 257-262.

[3] N.H. Sweilam and M.M. Khader, Exact solutions of some coupled nonlinear partial differential equations using the homotopy perturbation method, Computers \& Mathematics with Applications, 58 (2009), 2134-2141.

[4] J. Saberi-Nadjafi and A. Ghorbani, He's homotopy perturbation method: an effective tool for solving nonlinear integral and integro-differential equations, Computers \& Mathematics with Applications, 58 (2009), 1345-1351.

[5] A.V. Karmishin, A.I. Zhukov and V.G. Kolosov, Methods of Dynamics Calculation and Testing for Thin-Walled Structures, Mashinostroyenie, Moscow, Russia, 1990.

[6] R. Hirota, Exact solutions of the Korteweg-de Vries equation for multiple collisions of solitons, Physical Review Letters, 27 (1971), 1192-1194.

[7] A.M. Wazwaz, On multiple soliton solutions for coupled KdV-mkdV equation, Nonlinear Science Letters A, 1 (2010), 289-296.

[8] G. Adomian, Solving Frontier Problems of Physics: The Decomposition Method, Kluwer Acad. Publ., Boston, 1994.

[9] G.C. Wu and J.H. He, Fractional calculus of variations in fractal spacetime, Nonlinear Science Letters A, 1 (2010), 281-287.

[10] J.H. He, Variational iteration method - a kind of nonlinear analytical technique: some examples, International Journal of Nonlinear Mechanics, 34 (1999), 699-708.

[11] J.H. He and X.H. Wu, Variational iteration method: new development and applications, Computers \& Mathematics with Applications, 54 (2007), 881-894.

[12] J.H. He, G.C. Wu and F. Austin, The variational iteration method which should be followed, Nonlinear Science Letters A, 1 (2009), 1-30.

[13] L.A. Soltani and A. Shirzadi, A new modification of the variational iteration method, Computers \& Mathematics with Applications, 59 (2010), 2528-2535.

[14] N. Faraz, Y. Khan and A. Yildirim, Analytical approach to two-dimensional viscous flow with a shrinking sheet via variational iteration algorithm-II, Journal of King Saud University, 23 (2011), 77-81.

[15] G.C. Wu and E.W.M. Lee, Fractional variational iteration method and its application, Physics Letters A, 374 (25) (2010), 2506-2509.

[16] E. Hesameddini and H. Latifizadeh, Reconstruction of variational iteration algorithms using the Laplace transform, International Journal of Nonlinear Sciences and Numerical Simulation, 10 (2009), 1377-1382. 


\section{JAGDEV SINGH, DEVENDRA KUMAR AND SUSHILA}

[17] C. Chun, Fourier-series-based variational iteration method for a reliable treatment of heat equations with variable coefficients, International Journal of Nonlinear Sciences and Numerical Simulation, 10 (2009), $1383-1388$.

[18] D.J. Evans and H. Bulut, A new approach to the gas dynamics equation: An application of the decomposition method, Appl. Comput. Math., 79 (7) (2002), 817-822.

[19] H. Jafari, M. Zabihi and M. Saidy, Application of homotopy perturbation method for solving gas dynamics equation, 2 (48) (2008), 2393-2396.

[20] A. Ghorbani and J. Saberi-Nadjafi, He's homotopy perturbation method for calculating adomian polynomials, International Journal of Nonlinear Sciences and Numerical Simulation, 8 (2007), 229-232.

[21] A. Ghorbani, Beyond adomian's polynomials: He polynomials, Chaos Solitons Fractals, 39 (2009), $1486-1492$.

[22] S.T. Mohyud-Din, M.A. Noor and K.I. Noor, Traveling wave solutions of seventh-order generalized $\mathrm{KdV}$ equation using He's polynomials, International Journal of Nonlinear Sciences and Numerical Simulation, 10 (2009), 227-233.

[23] Y. Khan and Q. Wu, Homotopy perturbation transform method for nonlinear equations using He's polynomials, Computer and Mathematics with Applications, 61(8) (2011), 1963-1967.

[24] S.T. Mohyud-Din and A. Yildirim, Homotopy perturbation method for advection problems, Nonlinear Science Letters A, 1 (2010), 307-312.

[25] J.H. He, Homotopy perturbation method: a new nonlinear analytical technique, Applied Mathematics and Computation, 135 (2003), 73-79.

[26] J.H. He, Comparison of homotopy perturbation method and homotopy analysis method, Applied Mathematics and Computation, 156 (2004), 527-539.

[27] J.H. He, The homotopy perturbation method for nonlinear oscillators with discontinuities, Applied Mathematics and Computation, 151 (2004), 287-292.

[28] J.H. He, Homotopy perturbation method for bifurcation of nonlinear problems, International Journal of Nonlinear Sciences and Numerical Simulation, 6 (2005), 207-208.

[29] J.H. He, Some asymptotic methods for strongly nonlinear equation, International Journal of Modern Physics, 20 (2006), 1144-1199.

[30] J.H. He, Homotopy perturbation method for solving boundary value problems, Physics Letters A, 350 (2006), 87-88.

[31] M. Rafei and D.D. Ganji, Explicit solutions of helmhotz equation and fifth-order KdV equation using homotopy perturbation method, International Journal of Nonlinear Sciences and Numerical Simulation, 7 (2006), 321-328.

[32] A.M. Siddiqui, R. Mahmood and Q.K. Ghori, Thin film flow of a third grade fluid on a moving belt by He's homotopy perturbation method, International Journal of Nonlinear Sciences and Numerical Simulation, 7 (2006), 7-14.

[33] D.D. Ganji, The applications of He's homotopy perturbation method to nonlinear equation arising in heat transfer, Physics Letters A, 335 (2006), 337-341.

[34] L. Xu, He's homotopy perturbation method for a boundary layer equation in unbounded domain, Computers \& Mathematics with Applications, 54 (2007), 1067-1070.

[35] J.H. He, An elementary introduction of recently developed asymptotic methods and nanomechanics in textile engineering, International Journal of Modern Physics, 22 (2008), 3487-4578.

[36] J.H. He, Recent developments of the homotopy perturbation method, Topological Methods in Nonlinear Analysis, 31 (2008), 205-209.

[37] E. Hesameddini and H. Latifizadeh, An optimal choice of initial solutions in the homotopy perturbation method, International Journal of Nonlinear Sciences and Numerical Simulation, 10 (2009), 1389-1398.

[38] E. Hesameddini and H. Latifizadeh, A new vision of the He's homotopy perturbation method, International Journal of Nonlinear Sciences and Numerical Simulation, 10 (2009), 1415-1424.

[39] J. Biazar, M. Gholami Porshokuhi and B. Ghanbari, Extracting a general iterative method from an adomian decomposition method and comparing it to the variational iteration method, Computers \& Mathematics with Applications, 59 (2010), 622-628.

[40] S.A. Khuri, A Laplace decomposition algorithm applied to a class of nonlinear differential equations, Journal of Applied Mathematics, 1 (2001), 141-155.

[41] E. Yusufoglu, Numerical solution of Duffing equation by the Laplace decomposition algorithm, Applied Mathematics and Computation, 177 (2006), 572-580.

[42] Yasir Khan, An effective modification of the Laplace decomposition method for nonlinear equations, International Journal of Nonlinear Sciences and Numerical Simulation, 10 (2009), 1373-1376.

[43] Yasir Khan and Naeem Faraz, A new approach to differential difference equations, Journal of Advanced Research in Differential Equations, 2 (2010), 1-12.

[44] S. Islam, Y. Khan, N. Faraz and F. Austin, Numerical solution of logistic differential equations by using the Laplace decomposition method, World Applied Sciences Journal, 8 (2010), 1100-1105. 
[45] M. Madani and M. Fathizadeh, Homotopy perturbation algorithm using Laplace transformation, Nonlinear Science Letters A, 1 (2010), 263-267.

[46] S.T. Mohyud-Din, M.A. Noor, K.I. Noor, Traveling wave solutions of seventh-order generalized KdV equation using He's polynomials, International Journal of Nonlinear Sciences and Numerical Simulation, 10 (2009), 227-233.

[47] M.A. Noor, S.T. Mohyud-Din, Variational homotopy perturbation method for solving higher dimensional initial boundary value problems, Mathematical Problems in Engineering 2008 (2008) 11. Article ID 696734, doi:10.1155/2008/696734.

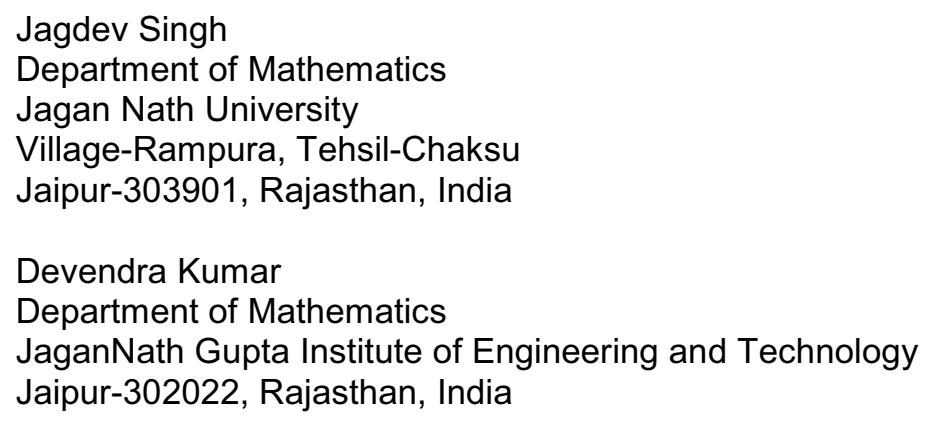

\section{Sushila \\ Department of Physics \\ Jagan Nath University \\ Village-Rampura, Tehsil-Chaksu \\ Jaipur-303901, Rajasthan, India}

Received July 2011 